\title{
A Regularized and Renormalized Electrostatic Coupling Hamiltonian for hybrid Quantum-Mechanical-Molecular- Mechanical Calculations
}

\author{
Pradip K. Biswas \\ Cleveland State University, pbiswas@tougaloo.edu \\ Valentin Gogonea \\ Cleveland State University, V.GOGONEA@csuohio.edu
}

Follow this and additional works at: https://engagedscholarship.csuohio.edu/scichem_facpub

Part of the Chemistry Commons

How does access to this work benefit you? Let us know!

\section{Publisher's Statement}

(c) 2005 American Institute of Physics.

\section{Recommended Citation}

Biswas, Pradip K. and Gogonea, Valentin, "A Regularized and Renormalized Electrostatic Coupling Hamiltonian for hybrid Quantum-Mechanical-Molecular-Mechanical Calculations" (2005). Chemistry Faculty Publications. 300.

https://engagedscholarship.csuohio.edu/scichem_facpub/300

This Article is brought to you for free and open access by the Chemistry Department at EngagedScholarship@CSU. It has been accepted for inclusion in Chemistry Faculty Publications by an authorized administrator of EngagedScholarship@CSU. For more information, please contact library.es@csuohio.edu. 
A regularized and renormalized electrostatic coupling Hamiltonian for hybrid quantummechanical-molecular-mechanical calculations

P. K. BiswasV. Gogonea

Citation: The Journal of Chemical Physics 123, 164114 (2005); doi: 10.1063/1.2064907

View online: http://dx.doi.org/10.1063/1.2064907

View Table of Contents: http://aip.scitation.org/toc/jcp/123/16

Published by the American Institute of Physics

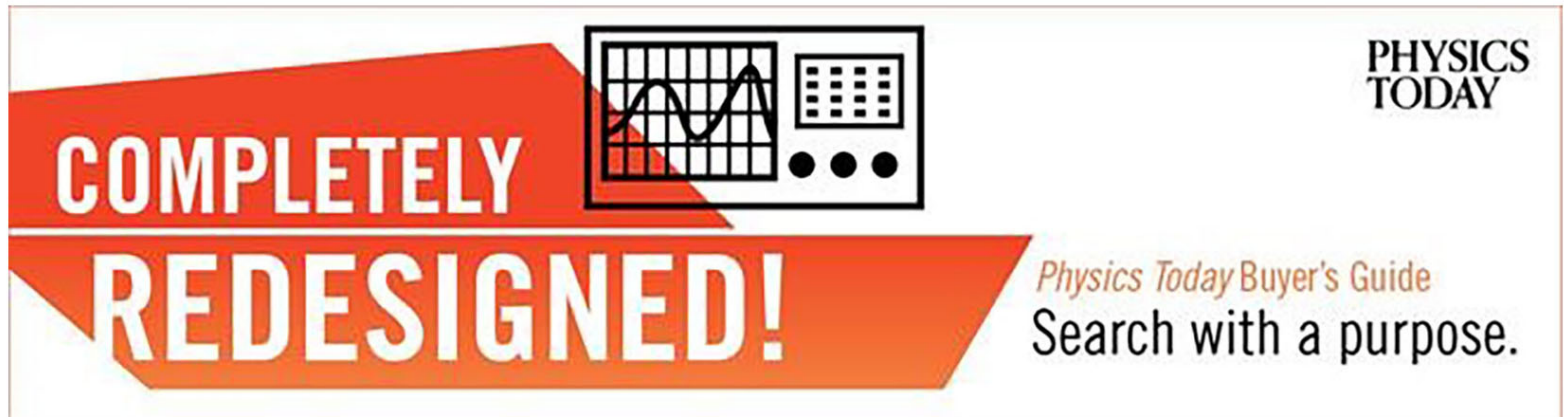




\title{
A regularized and renormalized electrostatic coupling Hamiltonian for hybrid quantum-mechanical-molecular-mechanical calculations
}

\author{
P. K. Biswas \\ Department of Chemistry, Cleveland State University, Cleveland, Ohio 44115 \\ V. Gogonea \\ Department of Chemistry, Cleveland State University, Cleveland, Ohio 44115 \\ and Cleveland Clinic Foundation, Cleveland, Ohio 44195
}

(Received 26 May 2005; accepted 22 August 2005; published online 27 October 2005)

\begin{abstract}
We describe a regularized and renormalized electrostatic coupling Hamiltonian for hybrid quantum-mechanical (QM)-molecular-mechanical (MM) calculations. To remedy the nonphysical QM/MM Coulomb interaction at short distances arising from a point electrostatic potential (ESP) charge of the MM atom and also to accommodate the effect of polarized MM atom in the coupling Hamiltonian, we propose a partial-wave expansion of the ESP charge and describe the effect of a $s$-wave expansion, extended over the covalent radius $r_{c}$, of the MM atom. The resulting potential describes that, at short distances, large scale cancellation of Coulomb interaction arises intrinsically from the localized expansion of the MM point charge and the potential self-consistently reduces to $1 / r_{c}$ at zero distance providing a renormalization to the Coulomb energy near interatomic separations. Employing this renormalized Hamiltonian, we developed an interface between the Car-Parrinello molecular-dynamics program and the classical molecular-dynamics simulation program Groningen machine for chemical simulations. With this hybrid code we performed QM/ MM calculations on water dimer, imidazole carbon monoxide (CO) complex, and imidazole-heme$\mathrm{CO}$ complex with $\mathrm{CO}$ interacting with another imidazole. The QM/MM results are in excellent agreement with experimental data for the geometry of these complexes and other computational data found in literature. (C) 2005 American Institute of Physics. [DOI: 10.1063/1.2064907]
\end{abstract}

\section{INTRODUCTION}

Atomistic simulation for structure calculation and the description of chemical reactions in complex systems, in particular, in supramolecular biological systems possess great challenges in computational biology and biological chemistry. ${ }^{1}$ While molecular-mechanical (MM) models with a classical force-field description for the interactions between the atoms are capable of providing a fairly accurate atomistic simulation of a complex system revealing its optimized geometry, vibrational frequency, etc., it falls short of describing a chemical reaction which involves the participation of valence electrons of the atoms concerned. Quantummechanical (QM) calculations, on the other hand, are capable of providing all the above-mentioned information including the chemical reactions. However, the computational cost grows with $N^{4}$ ( $N \equiv$ number of atoms) in an ab initio QM calculation and limits its application to small systems (a few hundred atoms) only (except for linear-scaling methods that implement special techniques). ${ }^{2}$ Density-functional theory ${ }^{3}$ (DFT) has become the method of choice for the electronic structure calculations and the description of reaction mechanisms in wide areas of chemical, biological, and solid-state systems owing its ability to deal with larger systems and the limitations of the $a b$ initio electronic many-body models. However, supramolecular biological systems, such as pro-

${ }^{a)}$ Electronic mail: v.gogonea@csuohio.edu teins, are too large to be described by a DFT model and the requirement on the size, accuracy, and duration of the simulation time is difficult to reconcile. ${ }^{4,5}$ Semiempirical methods such as modified neglect of differential overlap (MNDO), ${ }^{6}$ Austin model I (AMI) ${ }^{7}$ and third parametrization ${ }^{8}$ (PM3) are also in use to reconcile with the computational requirement. However, semiempirical methods are suitable only for systems for which parameters exist. ${ }^{9}$ Also there are bottlenecks regarding parametrization of elements. This has led to the culmination of hybrid QM/MM schemes ${ }^{10-15}$ where a complex molecular system is partitioned into chemically active and surrounding regions. The chemically active region is described quantum mechanically while the surrounding regions are described by MM force fields.

In principle, the QM subsystem can be described with any ab initio theory, DFT, or semiempirical method depending on the accuracy needed, availability of parameters for the semiempirical method, etc. But any QM/MM combination needs its appropriate coupling Hamiltonian to describe the interaction between the atoms in the QM and MM partitions. Development of an appropriate coupling Hamiltonian between the two subsystems, one in the QM part and the other in the MM part, is the biggest challenge in such a hybrid model. ${ }^{4,9,13}$ Depending on the partitioning, the QM/MM coupling Hamiltonian may have contribution from both bonded and nonbonded interactions. The QM/MM bonded interactions, which arise when the partition is made across cutting a chemical bond, face a different kind of challenge of saturat- 
ing the valence band for the QM subsystem and have been actively addressed in literature. ${ }^{13,16-22}$ The simplest solution is to add a monovalent link atom to saturate the valence band. ${ }^{16-18}$ The link atom concept has been further extended to double link atom method by Das et al. ${ }^{22}$ Other solutions involve the use of frozen orbital methods ${ }^{13,17,19-21}$ and the use of boundary atoms described by suitable pseudopotentials. ${ }^{23}$ The nonbonded interactions, on the other hand, face a different challenge. Due to pointlike description of the MM-atom charges and their interactions with the QM electrons at short ranges (much less than interatomic separations) can cause an artificial and nonphysical polarization of the QM electron density. ${ }^{4,9,22}$ Such an artificial polarization of the electron density around an active site can influence the outcome of a chemical reaction study, the dipole moment, and other properties calculated based on electronic charge density. ${ }^{4,9,22}$ The issue has been addressed in different ways $4,9,14,22$ and is the principal focus of this work.

As it stands, the electrostatic potential (ESP) point charge is meant for reproducing the electrostatic potential between atoms in a molecule where the atom-atom separation is of the order of an angstrom or above. It is basically a renormalization of the Coulomb potential at interatomic separations. However, for the QM/MM Hamiltonian, the separation between a point charge and QM electrons can take any value from zero to the size of the system. Obviously, the point-charge description for the MM atom cannot provide a compatible picture for the QM/MM Coulomb interaction at short distances nearing zero and is the source of the nonphysical polarization of QM electrons and divergent force on the MM atom. ${ }^{4}$ To remove this divergence, arising from a point-charge prescription for the $\mathrm{MM}$ atom, we propose a localized partial-wave expansion of the MM charge and adjust the extension of spatial distribution so that the Coulomb potential renormalizes near interatomic separations which is of the order of twice the covalent radius. Employing an $s$-wave expansion of the ESP charge, we show that this scheme naturally leads to large scale cancellations in the Coulomb potential at short distances and the potential intrinsically reduces to a value of $\sim 1 / r_{c}$ ( $r_{c}=$ covalent radius) thus avoiding nonphysical localization of QM electrons on a positively charged MM atom. The localized partial-wave expansion scheme for the ESP charge, which can include the effect of a polarized MM atom in the coupling Hamiltonian, and the derived analytical form of the Coulomb potential (see Appendix), which exhibits the regularization of the potential at short range and renormalization to the Coulomb energy at intermediate range, are the principal contributions of this work.

Employing the expansion scheme, we develop ${ }^{24}$ an interface between the MM program Groningen machine for chemical simulations $^{25}$ (GROMACS) version 3.2.1 and the density-functional-theory-based QM program Car-Parrinello molecular dynamics ${ }^{26}$ (CPMD) version 3.9.1. The following section describes the theoretical model in detail. In Sec. III, we discuss the results obtained employing the renormalized Hamiltonian.

\section{QM/MM HAMILTONIAN AND ITS COUPLING TO QM}

The central issue of a QM/MM hybridization scheme is the definition of the QM/MM coupling part $H_{\mathrm{QM} / \mathrm{MM}}$ of the total Hamiltonian $H$ given by ${ }^{9,11-13}$

$$
H=H_{\mathrm{QM}}+H_{\mathrm{MM}}+H_{\mathrm{QM} / \mathrm{MM}} .
$$

$H_{\mathrm{QM} / \mathrm{MM}}$ accounts for the interaction between the quantum system and the $\mathrm{MM}$ atoms. In general, $H_{\mathrm{QM} / \mathrm{MM}}$ contains Coulomb (long-range) and short-range interactions (van der Waals attraction and short-range repulsion) and is taken as ${ }^{9,11-13}$

$$
\begin{aligned}
H_{\mathrm{QM} / \mathrm{MM}}= & -\sum_{\frac{i \in \mathrm{QM}}{j \in \mathrm{MM}}} \frac{q_{j}}{\left|\mathbf{r}_{i}-\widetilde{\mathbf{R}}_{j}\right|}+\sum_{\frac{\nu \in \mathrm{QM}}{j \in \mathrm{MM}}} \frac{Z_{\nu} q_{j}}{\left|\mathbf{R}_{\nu}-\widetilde{\mathbf{R}}_{j}\right|} \\
& +\sum_{\frac{\nu \in \mathrm{QM}}{j \in \mathrm{MM}}} 4 \epsilon_{\nu j}\left\{\left(\frac{\sigma_{\nu j}}{\left|\mathbf{R}_{\nu}-\widetilde{\mathbf{R}}_{j}\right|}\right)^{12}-\left(\frac{\sigma_{\nu j}}{\left|\mathbf{R}_{\nu}-\widetilde{\mathbf{R}}_{j}\right|}\right)^{6}\right\},
\end{aligned}
$$

where $\mathbf{r}_{i}, \mathbf{R}_{\nu}$, and $\widetilde{\mathbf{R}}_{j}$ represent the position vector for the $i$ th electron, $\nu$ th $\mathrm{QM}$ nucleus with charge $Z_{\nu}$, and $j$ th $\mathrm{MM}$ atom with partial charge $q_{j}$, respectively. The short-range repulsion and attractive mutual average polarization (van der Waals) between QM and MM atoms have been modeled using the Lennard-Jones (LJ) potential; ${ }^{27} \sigma$ and $\epsilon$ are parameters defining the LJ potential. In the present state of the art of QM calculations nuclear and electronic motions are decoupled through the Born-Oppenheimer (BO) approximation. Thus, it is not possible for a $\mathrm{QM} / \mathrm{MM}$ calculation scheme to couple the van der Waals interaction between QM and MM atoms to the electronic motion of the QM system as the classical van der Waals interaction between two atoms is represented as a function of internuclear separations. However, the Coulomb interaction can be directly coupled, but the presence of point charges in the vicinity of the quantum system leads to artifacts ${ }^{4}$ in the results.

The central artifact that arises with the coupling of only the Coulomb interaction is that the electronic charge density of the QM system is poised to polarize nonphysically around the pointlike external ESP charges. ${ }^{4}$ The artifact is understood to have two allied sources. First, some of the QM electron density may find themselves quite close to the external point ESP charges; distances where the point ESP charge description is not valid and would create artifact. Second, in the absence of Pauli exclusion repulsion for the QM electrons by the MM atoms, the former would end up collapsing on a positive ESP charge. In reality, the atom treated by MM method with a point charge should have exerted Pauli repulsion due to its own electrons (which are replaced together with the nuclear charge by an effective point charge) and would deter the QM electrons to penetrate the atom valence shell. In a purely classical force-field calculation, the $1 /\left|\mathbf{R}_{\nu}-\widetilde{\mathbf{R}}_{j}\right|^{12}$ term of the Lennard-Jones potential ${ }^{27}$ supplements for this effect and provides sufficient repulsion between atoms at short range, thus keeping the attractively interacting MM atoms at appropriate separations. For QM theories, the Pauli exclusion repulsion is incorporated either 
properly antisymmetrizing the electronic wave function [for methods with Hatree-Fock (HF) origins] or by employing an exclusion hole concept (for methods with DFT origin). Nevertheless, incorporating the Pauli exclusion repulsion between the QM electronic charge distribution and the MM point charges in a hybrid $\mathrm{QM} / \mathrm{MM}$ calculation remains a formidable challenge and there is as such no prescription. In this communication, we seek a comprehensive description of the QM/MM Coulomb interaction considering a localized expansion of the ESP charges which regularizes the potential at short range while renormalizes to the Coulomb potential reminiscent to the ESP charge for larger distances $\left(r \geqslant 2 r_{c}\right)$. As the ESP charge reflects the overall electrostatic potential acting at a point in the configuration space, it accounts for the Pauli exclusion effect too. However, this conjecture is valid only beyond a certain radius and not at short distances where the notion of point ESP charge looses its validity. We attempt to regularize the potential at these short distances without affecting its value for distances $\geqslant 2 r_{c j}$.

Earlier, Eichinger et al. ${ }^{14}$ have described the MM point charge as a Gaussian delocalized charge density to remedy the short-range artifact. They used a multistep approach to evaluate the Coulomb interaction between a MM atom and the quantum system. In essence, their prescription of the Coulomb part of their hybrid QM/MM Hamiltonian is given by

$$
H_{\rho q_{j}}=N_{\rho} \int d^{3} r \rho(\mathbf{r}) q_{j} \frac{\operatorname{erf}\left(\left|\mathbf{r}-\widetilde{\mathbf{R}}_{j}\right| / \sigma\right)}{\left|\mathbf{r}-\widetilde{\mathbf{R}}_{j}\right|} .
$$

Here $\rho(\mathbf{r})$ is the electronic charge density at the grid point $\mathbf{r}$, $N_{\rho}$ is the normalization constant for the electronic charge density $\rho$, erf is the error function, $\widetilde{\mathbf{R}}_{j}$ is the position vector of the $j$ th MM atom, and the value of $\sigma$ is the same for all atoms $(0.8 \AA)$. As the error function (which integrates a Gaussian distribution over a certain radius) asymptotically reaches the value of unity, the above function has the correct asymptotic behavior for the Coulomb interaction at large distance. At short distances, the error function is less than unity and it tends to zero as distance goes to zero, thus removing the discontinuity in the QM/MM interaction potential. We compare the functional behavior of this form of the potential vis-a-vis the pure Coulomb interaction in Fig. 1. It appears that the potential does not saturate near twice the covalent radius of the atom, which is supposed to be a key issue in the modeling of the Coulomb QM/MM interaction.

Recently, Laio et al. ${ }^{4}$ have introduced another functional form that takes into account the short-range effect with the Coulomb potential saturating near the covalent radius of the MM atom. The Coulomb part of their hybrid QM/MM Hamiltonian is given by

$$
H_{\rho q_{j}}=N_{\rho} \int d^{3} r \rho(\mathbf{r}) \times q_{j} \frac{r_{c j}^{n}-\widetilde{r}^{n}}{r_{c j}^{n+1}-\widetilde{r}^{n+1}},
$$

where $\tilde{r} \equiv\left|\mathbf{r}-\widetilde{\mathbf{R}}_{j}\right|$. In the above prescription, the usual Coulomb interaction of $1 / \widetilde{r}$ is being replaced by a functional $v(\widetilde{r})=\left(r_{c j}^{n}-\widetilde{r}^{n}\right) /\left(r_{c j}^{n+1}-\widetilde{r}^{n+1}\right)$. This functional form also has the correct asymptotic behavior of $1 / \tilde{r}$ and as $\widetilde{r} \rightarrow 0$, it smoothly

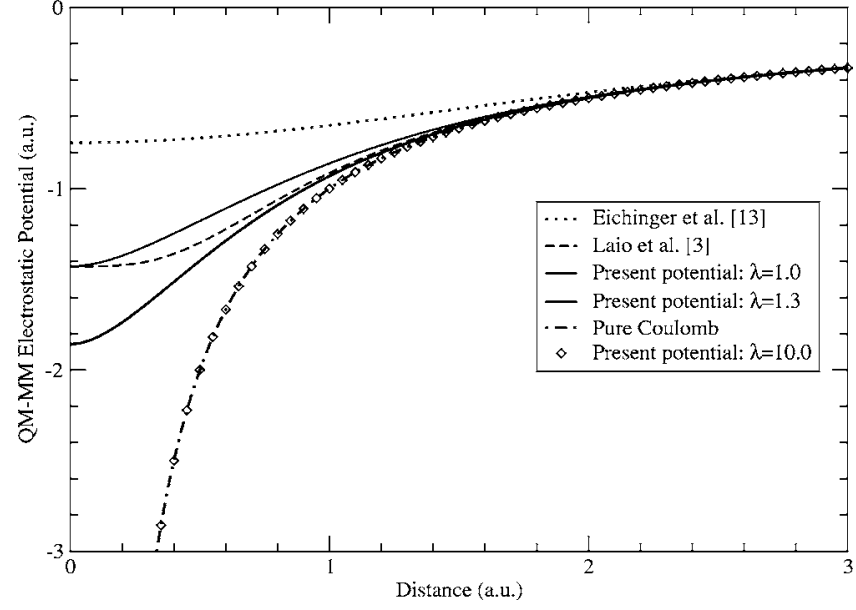

FIG. 1. Electrostatic interaction potential between an electron (a.u.) and a unit positive point charge representing a $\mathrm{MM}$ atom. Present potential refers to Eq. (A6) of the Appendix with $q_{j}=1.0$ a.u.

converges to the value provided by $1 / r_{c j}$. In Fig. 1 we show the behavior of the potential $v(\widetilde{r})$ with $r_{c j}=0.699$ a.u. (or $=0.37 \AA$ ). This corresponds to electrostatic potential of a $\mathrm{QM}$ electron with a unit positive ESP charge. The functional form, although appears very useful for QM/MM electrostatic interaction, has not been derived theoretically and thus may be considered as empirical. The functional forms of Eichinger et $a l .{ }^{14}$ and of Laio et al. ${ }^{4}$ mentioned above reduce both the attractive and repulsive Coulomb interactions at short distances while having the correct asymptotic behavior. Another crucial aspect of these prescriptions is that they lead to zero forces (finite potential) at very short ranges, thus avoiding the artificial localization of the electronic charge density on a positive MM point charge. Laio et al. ${ }^{4}$ also remarked that they were not successful in finding a functional form that provides repulsion at short distances and could mimic the Pauli exclusion between electronic charge density and the MM point charge. As mentioned by the authors, they have obtained good results for, e.g., $r_{c}(\mathrm{H}) \sim 0.4 \AA$ and $r_{c}(\mathrm{O}) \sim 0.7 \AA$, which are quite close to the accepted covalent radii used in this calculation.

Here, we seek a regularized and renormalized description for the QM/MM electrostatic interaction by arguing that the pointlike description of the ESP charge must be valid at interatomic separation (which is of the order of twice the covalent radius) but at short distances the Coulomb potential must be given by a localized charge distribution. To seek a comprehensive model for the QM/MM Coulomb interaction that could account for the short-range effect, we first consider a localized wave function $\varphi\left(\mathbf{r}^{\prime}-\widetilde{\mathbf{R}}_{j}\right)$ for the ESP charge present at $\widetilde{\mathbf{R}}_{j}$ so that the normalization of the wave function provides the charge $q_{j}$,

$$
\int\left|\varphi\left(\mathbf{r}^{\prime}-\widetilde{\mathbf{R}}_{j}\right)\right|^{2} d^{3} r^{\prime}=q_{j}
$$

where $r^{\prime}$ is an arbitrary point in space. For $\varphi$, we propose a partial-wave expansion in terms of an orthonornal basis set $\phi_{\ell m}\left(=\mathcal{R}_{\ell}(u) Y_{\ell m}(\hat{u})\right)$ of a hydrogen-like wave function and take 


$$
\varphi(\mathbf{u})=\left(\frac{q_{j}}{\sum_{\ell}\left|C_{\ell}\right|^{2}}\right)^{1 / 2} \sum_{\ell m} C_{\ell} \mathcal{R}_{\ell}(u) Y_{\ell m}(\hat{u}),
$$

where $\mathcal{R}_{\ell}(u)$ is similar to the radial part of the hydrogen-like wave function and $Y_{\ell m}(\hat{u})$, the spherical harmonics, represent the angular part; $C_{\ell}$ is the expansion coefficient. Similar partial-wave expansion to construct a wave function is frequently used in atomic molecular physics. ${ }^{28-30}$ In this work we adopt a first-order approximation $(\ell=0)$ to the expansion scheme which would allow us to account for the de-localized effect of the ESP charge in the $s$-wave approximation. The effect of polarized ESP charge, which may now be accounted with higher values of $\ell$, will be considered in our future communications. For a first-order approximation $(\ell=0)$ to the above expansion, we obtain

$$
\varphi\left(\mathbf{r}^{\prime}-\tilde{\mathbf{R}}_{j}\right)=\left(\frac{q_{j} \xi^{3}}{\pi}\right)^{1 / 2} e^{-\xi\left|\mathbf{r}^{\prime}-\tilde{\mathbf{R}}_{j}\right|}
$$

The Slater function provides a consistent picture with the localized description of an ESP charge and also enables us to arrive at analytical forms for the potential and force as shown below for the $s$ wave. A similar expansion scheme, but using Gaussian orbitals, has been employed earlier by Das et al. ${ }^{22}$ to study QM/MM systems. Although both the Gaussian and Slater orbitals are known to provide competitive results, the Slater orbitals have the proper behavior (cusp) at the origin while the Gaussian orbitals are generally easier to deal with computationally. However, the analytical form obtained here for the Coulomb potential [vide infra Eq. (10)] using Slater orbitals provides the same computational advantage as Gaussian orbitals. Here the parameter $\xi$ of the Slater orbital has the dimension of (length) ${ }^{-1}$ and we associate it to the reciprocal of covalent radius $r_{c j}: \xi \sim 1 / r_{c j}$. We write $\xi$ $=\lambda / r_{c j}$, where the parameter $\lambda$ will be used to renormalize the Coulomb energy at about $2 r_{c j}$ (the interatomic separation). $\lambda$ controls the spread of the wave function, and for $\lambda \gg 1$ the charge distribution collapses to a pointlike charge.

With the above wave-function description for the ESP charge, we now describe the Coulomb interaction potential (static potential) between the $j$ th $\mathrm{MM}$ atom and the QM system as

$$
\begin{aligned}
& H_{\rho q_{j}}=N_{\rho} \int d^{3} r \int d^{3} r^{\prime} \frac{\rho(\mathbf{r}) \mid \varphi\left(\mathbf{r}^{\prime}-\left.\widetilde{\mathbf{R}}_{j}\right|^{2}\right.}{\left|\mathbf{r}^{\prime}-\mathbf{r}\right|}, \\
& H_{Z_{\nu} q_{j}}=\sum_{\nu \in \mathrm{QM}} \int d^{3} r^{\prime} \frac{Z_{\nu}\left(\mathbf{R}_{\nu}\right)\left|\varphi\left(\mathbf{r}^{\prime}-\widetilde{\mathbf{R}}_{j}\right)\right|^{2}}{\left|\mathbf{r}^{\prime}-\mathbf{R}_{\nu}\right|},
\end{aligned}
$$

where $H_{\mathrm{QM} / \mathrm{MM}}^{\mathrm{Coul}}=H_{\rho q_{j}}+H_{Z_{\nu} q_{j}} ; Z_{\nu}$ is the charge of the ionic core of the $\nu$ th QM atom (i.e., sum of the nuclear and inner electron charges). In CPMD the ionic cores can be distributed over the grid used also by electronic charge density. But this way of distributing ionic core charges would not lead to any appreciable modification to the Coulomb energy as the separation between the $\mathrm{QM}$ nuclei and the $\mathrm{MM}$ atom is of the order of interatomic separation in a molecule and thus would be quite compatible with the point-charge description. Thus, here we focus on the effect of the spatial distribution of the
MM charges on the QM electron density. After performing the integrals over $d^{3} r^{\prime}$, as shown in the Appendix, we arrive at the following analytic expressions:

$$
\begin{aligned}
H_{\rho q_{j}}= & N_{\rho} \int d^{3} r \rho(\mathbf{r}) q_{j}\left[\frac{1}{\left|\mathbf{r}-\widetilde{\mathbf{R}}_{j}\right|}-\frac{e^{-2 \xi\left|\mathbf{r}-\tilde{\mathbf{R}}_{j}\right|}}{\left|\mathbf{r}-\widetilde{\mathbf{R}}_{j}\right|}-\xi e^{-2 \xi\left|\mathbf{r}-\tilde{\mathbf{R}}_{j}\right|}\right], \\
H_{Z_{\nu} q_{j}} & =\sum_{\nu \in \mathrm{QM}} Z_{\nu}\left(\mathbf{R}_{\nu}\right) q_{j}\left[\frac{1}{\left|\mathbf{R}_{\nu}-\tilde{\mathbf{R}}_{j}\right|}-\frac{e^{-2 \xi\left|\mathbf{R}_{\nu}-\tilde{\mathbf{R}}_{j}\right|}}{\left|\mathbf{R}_{\nu}-\tilde{\mathbf{R}}_{j}\right|}\right. \\
& \left.-\xi e^{-2 \xi\left|\mathbf{R}_{\nu}-\tilde{\mathbf{R}}_{j}\right|}\right] .
\end{aligned}
$$

From the above, we see that asymptotically (i.e., for $\left.\left|\mathbf{r}-\widetilde{\mathbf{R}}_{j}\right| \rightarrow \infty\right), \quad H_{\rho q_{j}}$ converges to the Coulomb potential $1 /\left|\mathbf{r}-\widetilde{\mathbf{R}}_{j}\right|$. Also for $\xi \rightarrow \infty$ (which recovers the point-charge description of the MM charge), the expressions reduce to the usual Coulomb potential, as expected. At short distance, the effect of the localized distribution of the MM charge introduces large cancellation to the Coulomb interaction and leads to a finite potential given by $\xi$ ( $\xi$ has the dimension of $1 / r$ ). Thus this potential leads to zero forces as the distance approaches zero.

We found it interesting and worthwhile to mention that the empirical form of the Coulomb potential proposed by Laio et l. $^{4}$ [as given in Eq. (4)] provides a very similar behavior with the Coulomb potential developed in this work [Eq. (10)]; they differ marginally only at low and intermediate ranges, however, both potentials converge to the value of $1 / r_{c j}$ at zero distance. As the value of the parameter $\lambda$ is increased, one gradually approaches towards a point-charge description for the MM atom. In Fig. 1, we plot the present electrostatic potential for $\lambda=1.0,1.3$, and 10.0. We see that for $\lambda=10.0$ the electrostatic potential follows the usual Coulomb interaction until very short ranges. Later, in Sec. III, we would see how in this case the QM electron density is artificially polarized on a positively charged $\mathrm{MM}$ atom. At $0.97 \AA$ (1.83 a.u.-typical $\mathrm{H}-\mathrm{O}$ separation in water) the value of the electrostatic potential of Eichinger et al. ${ }^{14}$ Laio et al., ${ }^{4}$ and the present one [Eq. (10)] differs from the Coulomb potential arising from the point-charge description by about $9.5 \%, 1.3 \%$, and $1.9 \%$, respectively; all are smaller than the Coulomb potential of a point charge. This is to emphasize that for $\lambda=1.3$, the Coulomb potential approaches the point-charge potential faster and normalizes near $1.4 \mathrm{a}$.u. ( $0.74 \AA$ - the interatomic separation in hydrogen molecule). Our results show that a value of $\lambda=1.3$ reduces the above difference of $1.9 \%$ (obtained with $\lambda=1.0$ ) to $0.5 \%$ and the corresponding expansion of the ESP charge provides the best results for the geometry optimizations performed here [using the 1-Broyden-Fletcher-Goldfarb-Shanno ${ }^{31}$, (BFGS) algorithm as implemented in GROMACS (Ref. 25). So, the empirical form of Laio et al. as given in Eq. (4) describes the localized distribution of the MM point charge quite effectively and provides an understanding of the importance of accounting the smearing effect of the MM charge. As com- 
pared with the functional form of Laio et $a{ }^{4}{ }^{4}$ and with the present potential obtained with Slater orbital, the potential arising from a Gaussian distribution of the point charge ${ }^{14}$ as given in Eq. (3) overestimates this effect by about $46 \%$. In Sec. III we provide pictures of electron localization function $^{32}$ for different values of $\lambda$ demonstrating the possibility of nonphysical localization of the QM electrons for arbitrary Coulomb potential at short range (e.g., for $\lambda=10.0$ ).

From the point of view that the modification in the Coulomb potential reflects a de-localized effect of the MMatom ESP charge, both the QM electron density and the QM ionic cores should experience the same modified Coulomb potential. However, it seems that Laio et al. ${ }^{4}$ do not consider the smearing effect of the MM charge while calculating the interaction with the ionic cores (see Sec. IV of Ref. 4) and thus replaced the modified Coulomb potential with pure Coulomb interaction $1 /\left(\left|\mathbf{R}_{\nu}-\widetilde{\mathbf{R}}_{j}\right|\right)$. Although, at interatomic distances, the effect of the regularization arising through the smearing effect would be minimal, their neglect may lead to some inconsistency in the conservation of forces between electrons and nuclei (ionic core here). In our work we ensure that both the QM electrons and the ionic cores experience the same external potential.

\section{RESULTS AND DISCUSSIONS}

To describe the performance of the GROMACS-CPMD (Ref. 33) QM/MM method vis-a-vis the electrostatic coupling, we consider the examples of (i) a water dimer, (ii) the imidazole-carbon monoxide (CO) complex, and (iii) a heme group interacting with $\mathrm{CO}$. We start the description of the results with the water dimer, where the hydrogen atom of one water molecule forms a hydrogen bond with the oxygen atom of the other molecule. This provides a crucial test for the QM/MM interaction Hamiltonian as it takes the leading role in the formation of the hydrogen bond between two molecules, with one molecule treated by QM and the other by MM. The structure of the water dimer is known fairly accurately from experimental observations ${ }^{34}$ and the system has also been studied using various calculational schemes (see Ref. 14 and references therein).

For an isolated water molecule, the calculated $\mathrm{O}-\mathrm{H}$ length is $0.9584 \AA$ and the $\mathrm{H}-\mathrm{O}-\mathrm{H}$ angle is $104.474^{\circ} .{ }^{35}$ In water, the formation of hydrogen bond weakens the covalent bonding and neutron diffraction ${ }^{36}$ shows that the $\mathrm{O}-\mathrm{H}$ length and the $\mathrm{H}-\mathrm{O}-\mathrm{H}$ angle change to $0.97 \AA$ and $106^{\circ}$, respectively. To perform QM/MM calculation on the water dimer with one of the water molecules in the MM region, we need appropriate force-field parameters (point charges, bond stretching, angle bending constants, and van der Waals interaction) for water molecule which are capable of producing a fairly accurate structure of the water dimer if calculated by MM only. Thus, first we perform QM calculations for a single water molecule using GAUSSIAN $98^{37}$ [B3LYP with 6-311+G( $(d, p)$ basis set] and CPMD (Ref. 26) (Goedecker pseudopotential with BLYP and 120 Ry cutoff for the plane waves) to obtain fairly accurate partial charges derived from ESP. Accordingly, we set the ESP charges for the oxygen and hydrogen atoms to -0.786 and 0.393 a.u., respectively. The
TABLE I. Results for the geometry of a water dimer.

\begin{tabular}{|c|c|c|c|c|}
\hline Results & $d_{\mathrm{H} \text { bond }}$ & $d_{\mathrm{OO}}$ & $\angle \alpha$ & $\angle \beta$ \\
\hline Expt. $^{\mathrm{a}}$ & $\ldots$ & 2.976 & $6^{\circ} \pm 20^{\circ}$ & $57^{\circ} \pm 10^{\circ}$ \\
\hline G98[MP4] & 1.981 & 2.942 & $0.6^{\circ}$ & $58.1^{\circ}$ \\
\hline G98[MP2 $]^{\mathrm{c}}$ & 1.948 & 2.913 & $2.3^{\circ}$ & $51.0^{\circ}$ \\
\hline G98[B3LYP $]^{\mathrm{d}}$ & 1.933 & 2.900 & $3.5^{\circ}$ & $54.1^{\circ}$ \\
\hline G98[LDA $]^{\mathrm{e}}$ & 1.727 & 2.707 & $5.6^{\circ}$ & $61.7^{\circ}$ \\
\hline CPMD $[M T-B L Y P]^{\mathrm{f}}$ & 1.993 & 2.972 & $1.0^{\circ}$ & $55.7^{\circ}$ \\
\hline $\mathrm{CPMD}[\mathrm{SG}-\mathrm{LDA}]^{\mathrm{g}}$ & 1.737 & 2.727 & $1.0^{\circ}$ & $56.2^{\circ}$ \\
\hline GROMACS-CPMD[MT-BLYP $]^{\mathrm{h}}$ & 1.980 & 2.958 & $0.6^{\circ}$ & $69.3^{\circ}$ \\
\hline GROMACS-CPMD $[\mathrm{SG}-\mathrm{LDA}]^{\mathrm{i}}$ & 1.963 & 2.940 & $2.6^{\circ}$ & $66.1^{\circ}$ \\
\hline EGO-CPMD[MT-BLYP] $]^{j}$ & $\cdots$ & 2.850 & $\cdots$ & $63.0^{\circ}$ \\
\hline EGO-CPMD $[\text { MT-LDA }]^{\mathrm{k}}$ & $\cdots$ & 2.800 & $\cdots$ & $60.0^{\circ}$ \\
\hline GROMACS $^{1}$ & 2.010 & 3.000 & $0.0^{\circ}$ & $55.8^{\circ}$ \\
\hline
\end{tabular}

${ }^{a}$ Experimental (Ref. 34).

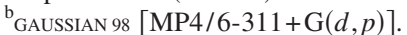

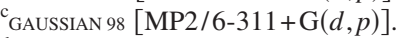

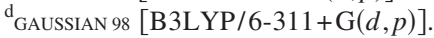

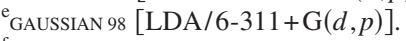

${ }_{\text {CPMD }}$ [MT-BLYP].

${ }^{\mathrm{C}} \mathrm{CPMD}[\mathrm{SG}-\mathrm{LDA}]$.

${ }^{\mathrm{h}}$ Present QM/MM: GROMACS-CPMD[MT-BLYP].

${ }^{\mathrm{i}}$ Present QM/MM: GROMACS-CPMD[SG-LDA].

${ }^{\mathrm{j}}$ Reference 14 QM/MM: EGO-CPMD[MT-BLYP].

${ }^{\mathrm{k}}$ Reference 14 QM/MM: EGO-CPMD[MT-LDA].

${ }^{\mathrm{l}}$ Pure Gromacs (Ref. 25) with opls force field (Ref. 43) as implemented in GROMACS.

Lennard-Jones parameters for the van der Waals potential for oxygen and hydrogen atoms were initially taken from the TIP3P model of water, ${ }^{38}$ however, we find that a slightly higher value of $\sigma=3.25061 \AA$ compared to $3.15061 \AA$ leads to a better structure for the water dimer. To judge the performance of the QM/MM interaction Hamiltonian, we calculate the optimized geometry for the water dimer without making any further adjustment to the classical force-field parameters (ESP charge, bond stretching, and angle bending).

In Table I we provide computational results for the water dimer employing QM/MM and compare them with full QM, $\mathrm{MM}$, and other $\mathrm{QM} / \mathrm{MM}$ calculations and experimental data. ${ }^{34}$ Table I shows that for fully QM calculations there is a consistent improvement of structural results when more sophisticated approximations of $a b$ initio theory are used. The results from both the GAUSSIAN 98 and CPMD codes using DFT are quite similar and consistent with each other. For fully MM calculations, the structural results are also consistent with the measured data. It is to be mentioned here that the angles $\alpha$ and $\beta$ (see Fig. 2), which are consequences of the interactions of the donor hydrogen atom with the lone

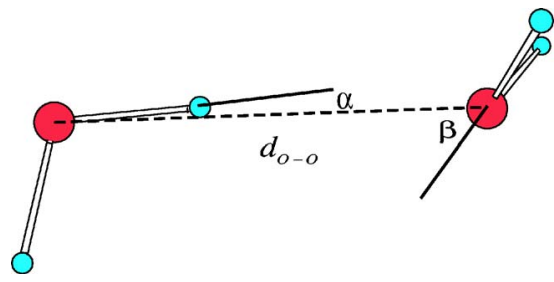

FIG. 2. Geometrical features of water dimer. 


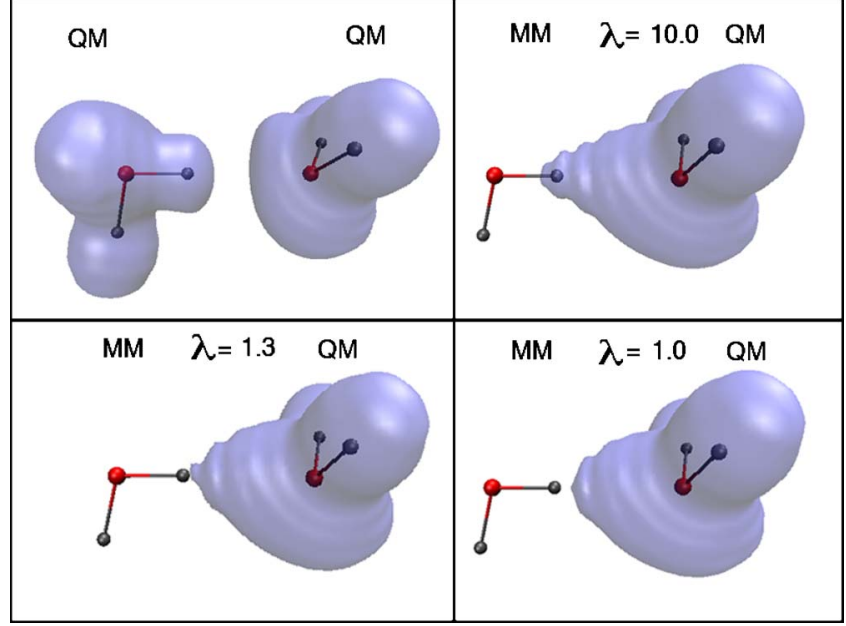

FIG. 3. Plot of electron localization function (ELF) for a water dimer as obtained from (a) a full quantum calculation employing CPMD[MT-BLYP] (Ref. 26), and a QM/MM calculation employing GROMACS-CPMD[MT-BLYP] with (b) $\lambda=10.0$, (c) $\lambda=1.3$, and (d) $\lambda=1.0$.

pair electrons of the acceptor oxygen, have no analog in the TIP3P-type water model, and these angles are basically simulated by the carefully adjusted ESP charges of the atoms containing the donor hydrogen. Table I lists the QM/MM results for two different combinations of DFT functionals and pseudopotentials. CPMD/GROMACS calculations with both types of pseudopotentials, Troullier-Martins ${ }^{39}$ (MT) and Goedecker $^{40}(\mathrm{SG})$, give reasonable results for the structural features of water dimer. BLYP exchange-correlation functional with the MT pseudopotential gives more accurate bond distances. It is worth noting that CPMD/GROMACS calculations give better results for the hydrogen bond and for the oxygen-oxygen distance compared to those of fully QM calculation with a localized density approximation (LDA) for exchange. The better results from QM/MM calculation implies that the present QM/MM electrostatic coupling Hamiltonian represents a better approximation for the interface Hamiltonian compared to that of a LDA Hamiltonian for the same interaction. Comparing with the EGO-CPMD QM/MM results, ${ }^{14}$ for the LDA and BLYP exchange correlation, we understand that with a better exchange correlation (BLYP) the angle $\beta$ increases by $5 \%-7 \%$. The variation in the angle $\beta$ in the calculation of water dimer has been discussed in detail by Mahoney and Jorgensen. ${ }^{41}$

To visualize this nonphysical polarization of the QM electron density around a positive ESP charge, we employ the CPMD utility program CPMD2CUBE and plot the electron localization function $^{32}$ (ELF) both for full QM and QM/MM calculations. The cubefiles ${ }^{37}$ are then visualized ${ }^{23}$ employing the visual molecular-dynamics (vMD) program. ${ }^{42}$. In Fig. 3, we provide a display of the ELF for a water dimer as obtained from a fully QM calculation employing CPMD [MT-BLYP] and also from QM/MM calculations with various levels of smearing effect of the ESP charge (obtained through various values of $\lambda$ ). We see that for $\lambda=10.0$ [for which the electrostatic potential of Eq. (10) closely follows the Coulomb potential of the point charge] a significant amount of QM electron density from the oxygen lone pair

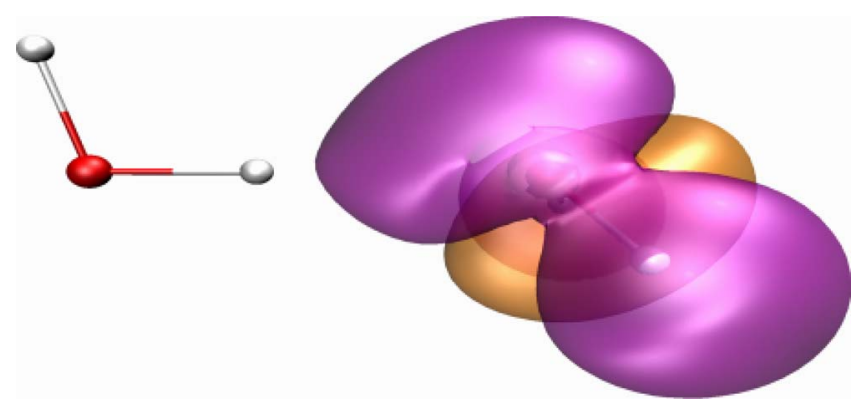

FIG. 4. Difference of electronic charge densities around the atoms of a water molecule in the QM system as obtained with QM/MM calculations employing GROMACS-CPMD[MT-BLYP] with external field of another water molecule with (a) the ESP charge is taken approximately as point charge $(\lambda \gg 1)$ and (b) the ESP charge is taken as a localized $s$-wave expansion with $\lambda=1.0$.

has the potential to be delocalized towards the donor hydrogen atom (which has a positive ESP charge). This effect is negligible for the case with $\lambda \sim 1.0$ as expected. To investigate the possibility of nonphysical delocalization with its entirety, we set the lower bound of the ELF isovalue to 0.6 in the generation of the VMD picture. For isovalues, of 0.8 or above, the nonphysical delocalization does not show up in the picture. This is to emphasize here that the amount and quality of nonphysical distortion do depend on the quality of pseudopotential used for the DFT model. The MT pseudopotentials are more robust against nonphysical distortions.

Figure 4 shows the density difference of the QM water molecule obtained with QM/MM calculation for two different levels of perturbation: (1) a MM water molecule with approximate point-charge description $(\lambda=10.0)$, and (2) a MM water molecule with a localized $s$-wave expansion with $\lambda=1.0$. The picture demonstrates the difference in polarization of the QM charge density for the two different spreads $(\lambda=1.0$ and 10.0) of the ESP charge. In the picture, the orange color represents regions of lost electron density and the magenta represents areas where electron density is gained. We also see in this picture how the electron density of one of the lone pairs of the acceptor oxygen is dragged by the donor hydrogen.

In the QM/MM calculation on a water dimer, the water molecule that is in the QM system will exhibit the lone pair electrons while the water molecule that is in the MM system will not have any electronic description. Thus, it is imperative that for QM/MM calculations on water dimer, the acceptor oxygen must be in the QM system so that it can provide the necessary lone pairs in its electronic density distribution so as to create the hydrogen bond between the donor hydrogen and the acceptor oxygen. Otherwise, there would be a gross error in the prediction of the angle $\beta$ (see Table I of Ref. 14). So, we do not address QM/MM calculation with the reverse configuration of left-hand-side water molecule in the QM part and the right-hand-side molecule in the MM part.

To judge the performance of the electrostatic coupling Hamiltonian further, we now discuss another example of $\mathrm{QM} / \mathrm{MM}$ interaction but in a different chemical environment. We discuss about the system of imidazole interacting with carbon monoxide (ImCO). We describe the imidazole ring using MM with optimized parameters for liquid simulation 
TABLE II. Results for an imidazole-carbon monoxide (ImCO) complex.

\begin{tabular}{lcccc}
\hline \hline Results & $d_{\mathrm{H} \ldots \mathrm{O}}$ & $d_{\mathrm{O}-\mathrm{N}}$ & $d_{\mathrm{C}-\mathrm{N}}$ & $d_{\mathrm{C}-\mathrm{O}}$ \\
\hline G98[MP4] $^{\mathrm{a}}$ & 2.349 & 3.353 & 4.481 & 1.129 \\
G98[LDA] $^{\mathrm{b}}$ & 2.053 & 3.072 & 4.200 & 1.131 \\
CPMD[MT-BLYP] $^{\mathrm{c}}$ & 2.412 & 3.394 & 4.447 & 1.136 \\
CPMD[SG-LDA] $^{\mathrm{d}}$ & 2.077 & 3.089 & 4.218 & 1.136 \\
GROMACS-CPMD[MT-BLYP] $^{\mathrm{e}}$ & 2.319 & 3.329 & 4.461 & 1.135 \\
GROMACS-CPMD[SG-LDA] $^{\mathrm{f}}$ & 2.367 & 3.378 & 4.502 & 1.129 \\
EGO-CPMD[MT-BLYP] $^{\mathrm{g}}$ & 1.700 & $\ldots$ & $\ldots$ & $\ldots$ \\
GROMACs $^{\mathrm{h}}$ & 2.272 & 3.278 & 4.405 & 1.128 \\
\hline
\end{tabular}

a Gaussian $98[\mathrm{MP} 4 / 6-311+\mathrm{G}(d, p)]$.

${ }^{\mathrm{b}}$ Gaussian $98[\mathrm{LDA} / 6-311+\mathrm{G}(d, p)]$

${ }^{\mathrm{CPMD}}$ [MT-BLYP].

${ }^{\mathrm{d}}$ CPMD [SG-LDA].

${ }^{\mathrm{e}}$ Present QM/MM: GROMACS-CPMD[MT-BLYP].

${ }^{\mathrm{f}}$ Present QM/MM: GROMACS-CPMD[SG-LDA].

${ }^{\mathrm{g}} \mathrm{QM} / \mathrm{MM}$ : EGO-CPMD[MT-BLYP] (Ref. 14).

${ }^{\mathrm{h}}$ Pure Gromacs (Ref. 25) with opLs force field (Ref. 43).

(OPLS) force field ${ }^{43}$ and the CO using QM. A fully QM calculation provides positive ESP charge for the oxygen of $\mathrm{CO}$. However, a fully MM calculation cannot provide an appropriate geometry for the ImCO complex until the ESP charge of the oxygen is negative. Thus, together with the fully QM calculation, we also obtain the geometry with a fully MM calculation so as to get an appropriate description for the ESP charge needed for the MM and QM/MM calculations. To obtain an appropriate geometry for ImCO with MM calculations we adjust the ESP charges for carbon and oxygen as +0.25 and -0.25 a.u., respectively, and also tune the OPLS value of $\sigma$ of the LJ potential of carbon to $4.95 \AA$ from $3.45 \AA$. For QM/MM calculations, we use the same set of parameters as for MM optimization. Table II presents the results obtained with fully $\mathrm{QM}$, fully $\mathrm{MM}$, and the QM/MM with the GROMACS-CPMD calculation employing LDA and BLYP exchange correlation and other computational results found in literature. From Table II, we find that the structural results from the present $\mathrm{QM} / \mathrm{MM}$ calculation compare quite well with CPMD and the sophisticated GAUSSIAN 98 results [MP4/6-311+G(d,p)].

Finally, we discuss the results for QM/MM calculation in gas phase for imidazole interacting with iron-porphyrin

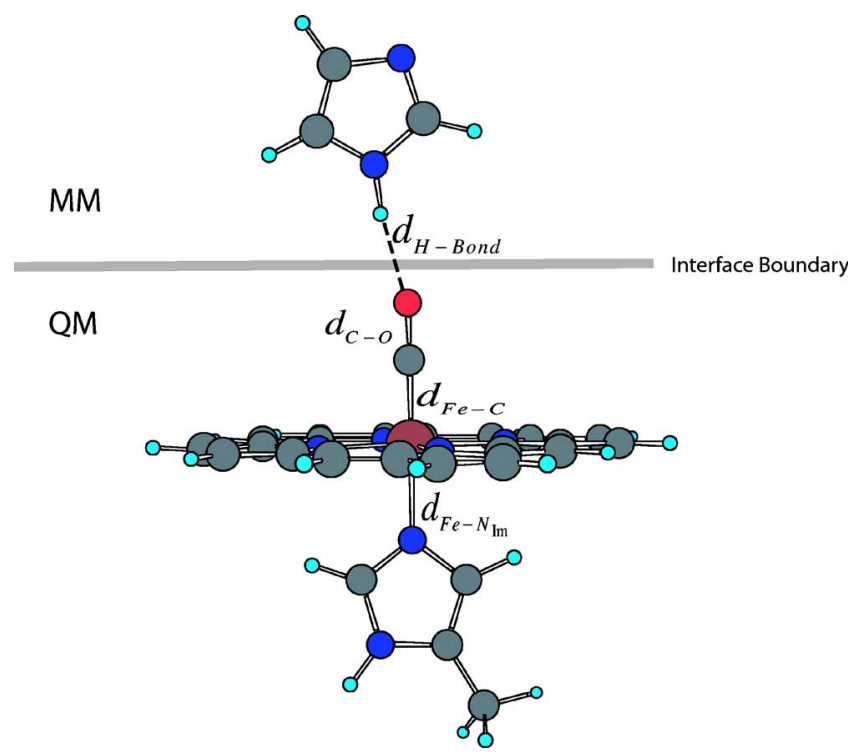

FIG. 5. Optimized geometry for $\mathrm{FeP}(\mathrm{Im})-\mathrm{CO}$ complex obtained with a QM/MM calculation employing CPMD (Ref. 26) and GROMACs (Ref. 25).

complex ligated with another imidazole and $\mathrm{CO}[\mathrm{FeP}(\mathrm{Im})-$ $\mathrm{CO}$. The $\mathrm{FeP}(\mathrm{Im})-\mathrm{CO}$ compound has been placed in the $\mathrm{QM}$ region while the second imidazole is being treated by MM. FeP(Im)-CO complex was put in the QM system because porphyrin is an extended electron delocalization system and OPLS parameters for this system that could be used with the OPLS force field were not available. We would like to point out that this is one of the advantages of a QM/MM method that it is not a hostage to the availability of accurate MM force-field parameters.

In Table III we provide our results for the gas phase QM/MM geometry optimization (shown in Fig. 5) for this system with the GROMACS-CPMD combination. The results are obtained with a cutoff of $25 \mathrm{Ry}$ for the wave function and $100 \mathrm{Ry}$ for the density, employing soft Vanderbilt (VDB) pseudopotentials. $^{44}$ We compare our results with the QM/MM optimized results of Rovira et al. ${ }^{5}$ obtained on different snapshots of classical MD simulations of solvated myoglobin and with gas phase calculation on $\mathrm{FeP}(\mathrm{Im})-\mathrm{CO}$ of Rovira. ${ }^{45} \mathrm{We}$ also compare the results with the experimental data on Fe(TPP)(py)-CO complex. ${ }^{46}$ Table III shows that

TABLE III. Results for the optimized geometry of an FeP(Im)-CO complex interacting with an imidazole.

\begin{tabular}{|c|c|c|c|c|c|c|}
\hline$\underline{\text { Results }}$ & $d_{\mathrm{H} \ldots \mathrm{O}}$ & $d_{\mathrm{Fe}-\mathrm{C}}$ & $d_{\mathrm{Fe}-N}$ & $d_{\mathrm{Fe}-N_{H}}$ & $d_{\mathrm{C}-\mathrm{O}}$ & $\angle \mathrm{Fe}-\mathrm{C}-\mathrm{O}$ \\
\hline GROMACS-CPMD $^{a}$ & 2.18 & 1.70 & $1.97-1.98$ & 1.99 & 1.17 & $178.9^{\circ}$ \\
\hline EGO-CPMD $(\mathrm{II})^{\mathrm{b}}$ & 2.69 & 1.74 & $1.99-2.02$ & 2.13 & 1.17 & $176.1^{\circ}$ \\
\hline EGO-CPMD $(\mathrm{III})^{\mathrm{b}}$ & 3.47 & 1.75 & $1.98-2.03$ & 2.10 & 1.16 & $179.3^{\circ}$ \\
\hline $\mathrm{Fe}(\mathrm{Im})-\mathrm{CO}^{\mathrm{c}}$ & $\cdots$ & 1.72 & 2.02 & 2.10 & 1.17 & $180.0^{\circ}$ \\
\hline $\mathrm{Fe}(\mathrm{TPP})\left(\right.$ py)-CO: Expt. ${ }^{\mathrm{d}}$ & $\cdots$ & 1.77 & 2.02 & 2.10 & 1.12 & $179.0^{\circ}$ \\
\hline Myoglobin-CO: Expt. ${ }^{\mathrm{e}}$ & $\cdots$ & 1.82 & 2.00 & 2.06 & 1.09 & $171.0^{\circ}$ \\
\hline
\end{tabular}

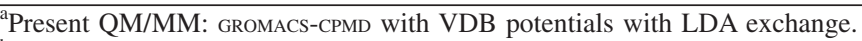

${ }^{\mathrm{b}}$ Results of Ref. 5 for EGO-CPMD for two different configurations.

${ }^{c}$ Results of Ref. 45 for FeP(Im)-CO.

${ }^{\mathrm{d}}$ Experimental results of Ref. 46 on Fe(TPP)(py)-CO.

${ }^{\mathrm{e}}$ Experimental results on Myogloblin-CO of Ref. 47. 
the H...O distance in the present calculation $(2.18 \AA)$ is in the range of a hydrogen bond while in the protein environment the distance is somewhat larger $(2.69 \AA)$ for a similar configuration of $\mathrm{CO}$ and imidazole ring [EGO-CPMD(II)]. This is probably due to the fact that imidazole is part of the His-64 residue and cannot move closer to $\mathrm{CO}$ as it is restrained in the protein environment. The minor differences in other distances, e.g. the $\mathrm{Fe}-\mathrm{N}_{p}$, are within $1 \%$ as compared with the results of Rovira ${ }^{45}$ and the $\mathrm{x}$-ray crystal structure data of myoglobin with heme ligated by carbon monoxide. ${ }^{47}$ These differences in the calculated results are supposed to be due to the use of different pseudopotentials, the cutoff in wave function and electron density, and the force-field parameters.

\section{SUMMARY}

In summary, we have developed a regularized and renormalized form for the electrostatic coupling Hamiltonian describing the interactions between atoms in the molecularmechanical (MM) and quantum-mechanical (QM) subsystems for a hybrid QM/MM calculation. The regularized and renormalized Hamiltonian arrived at considering an $s$-wave partial-wave expansion of the MM-atom ESP charge is found to remedy significantly the nonphysical distortions of the QM electron density arising for very short distances due to ESP point-charge description. Results obtained in this work for geometry optimization on water dimer, imidazoleCO complex, and FeP(Im)-CO-Im complex are in excellent agreement with experimental results and $a b$ initio quantum calculations. The plot of electron localization function (ELF) and density difference shows the importance of employing localized expansion for the ESP point charge in order to avoid unphysical polarization of QM electron density in hybrid QM/MM calculations. The localized wave expansion intrinsically removes the divergence in the Coulomb potential introduced by a point-charge description.

Contribution from higher partial waves $(p, d$, etc.) will allow us to incorporate the effect of polarized MM atoms in the coupling Hamiltonian. Works are in progress in this direction.

\section{ACKNOWLEDGMENTS}

We acknowledge the computational support from Kiran Tatapudi and Dr. Axel Kohlmeyer, and useful discussions with Dr. James Lock. This work was financially supported by the Department of Energy (Grant No. DE-FG0203ER15462) and the National Institutes of Health (Grant No. 1R15GM070469-01). Computational resources from the National Center for Supercomputer Applications (NCSA) at University of Illinois is also acknowledged.

\section{APPENDIX: DERIVATION OF COULOMB POTENTIAL FOR DELOCALIZED POINT CHARGES}

To perform the integration over $d^{3} r^{\prime}$ in Eq. (8), we pick up the $r^{\prime}$-dependent part

$$
I=\int d^{3} r^{\prime} \frac{\left|\varphi\left(\mathbf{r}^{\prime}-\widetilde{\mathbf{R}}_{j}\right)\right|^{2}}{\left|\mathbf{r}^{\prime}-\mathbf{r}\right|} .
$$

Substituting for $\varphi$ from Eq. (7) and taking Fourier transformations for $e^{-2 \xi\left|\mathbf{r}^{\prime}-\tilde{\mathbf{R}}_{j}\right|}$ and $1 /\left|\mathbf{r}^{\prime}-\mathbf{r}\right|$, we obtain

$$
\begin{aligned}
I= & \frac{q_{j} \xi^{3}}{\pi} \int d^{3} r^{\prime}\left(\frac{2 \xi}{\pi^{2}} \int d^{3} p \frac{e^{i \mathbf{p} \cdot\left(\mathbf{r}^{\prime}-\tilde{\mathbf{R}}_{j}\right)}}{\left(p^{2}+4 \xi^{2}\right)^{2}}\right) \\
& \times\left(\frac{1}{2 \pi^{2}} \int d^{3} q \frac{e^{i \mathbf{q} \cdot\left(\mathbf{r}^{\prime}-\mathbf{r}\right)}}{q^{2}}\right) .
\end{aligned}
$$

Rearranging and performing the integration over $d^{3} r^{\prime}$, we get

$$
\begin{aligned}
I= & \frac{q_{j} \xi^{3}}{\pi} \frac{2 \xi}{\pi^{2}} \frac{1}{2 \pi^{2}}(2 \pi)^{3} \int d^{3} p \int d^{3} q \delta(\mathbf{p}-\mathbf{q}) \\
& \times \frac{e^{-i \mathbf{p} \cdot \tilde{\mathbf{R}}_{j}}}{\left(p^{2}+4 \xi^{2}\right)^{2}} \frac{e^{i \mathbf{q} \cdot \mathbf{r}}}{q^{2}} .
\end{aligned}
$$

Performing the integration over $d^{3} q$ using $\delta$-function integration one obtains

$$
I=\frac{q_{j} \xi^{3}}{\pi} \frac{8 \xi}{\pi} \int d^{3} p \frac{e^{i \mathbf{p} \cdot\left(\mathbf{r}-\tilde{\mathbf{R}}_{j}\right)}}{p^{2}\left(p^{2}+4 \xi^{2}\right)^{2}} .
$$

Decomposing $1 /\left\{p^{2}\left(p^{2}+4 \xi^{2}\right)^{2}\right\}$ we rewrite the above integral as

$$
\begin{aligned}
I= & \frac{q_{j} \xi^{3}}{\pi} \frac{8 \xi}{\pi} \int d^{3} p\left[\frac{1}{\zeta^{4} p^{2}}-\frac{1}{\zeta^{4}\left(p^{2}+\zeta^{2}\right)}\right. \\
& \left.-\frac{1}{\zeta^{2}\left(p^{2}+\zeta^{2}\right)^{2}}\right] e^{i \mathbf{p} \cdot\left(\mathbf{r}-\tilde{\mathbf{R}}_{j}\right)},
\end{aligned}
$$

where $\zeta=2 \xi$. Taking inverse Fourier transforms for all the three integrals and simplifying for the constants, we finally obtain

$$
I=q_{j}\left[\frac{1}{\left|\mathbf{r}-\tilde{\mathbf{R}}_{j}\right|}-\frac{e^{-2 \xi\left|\mathbf{r}-\tilde{\mathbf{R}}_{j}\right|}}{\left|\mathbf{r}-\tilde{\mathbf{R}}_{j}\right|}-\xi e^{-2 \xi\left|\mathbf{r}-\tilde{\mathbf{R}}_{j}\right|}\right] .
$$

${ }^{1}$ T. C. Bruice and K. Kahn, Curr. Opin. Chem. Biol. 4, 540 (2000).

${ }^{2}$ G. Galli, Phys. Status Solidi B 217, 231 (2000).

${ }^{3}$ W. Koch and M. C. Holthausen, A Chemist's Guide to Density Functional Theory, 2nd ed. (Wiley, Weinheim, 2001).

${ }^{4}$ A. Laio, J. VandeVondele, and U. Rothlisberger, J. Chem. Phys. 116, 6941 (2002).

${ }^{5}$ C. Rovira, B. Schultze, M. Eichinger, J. D. Evanseck, and M. Parrinello, Biophys. J. 81, 435 (2001)

${ }^{6}$ M. J. S. Dewar and W. Thiel, J. Am. Chem. Soc. 99, 4899 (1977).

${ }^{7}$ M. J. S. Dewar, E. G. Zoebisch, E. F. Healy, and J. J. P. Stewart, J. Am. Chem. Soc. 107, 3902 (1985).

${ }^{8}$ J. J. P. Stewart, J. Comput. Chem. 10, 209 (1989).

${ }^{9}$ Y. Tu and A. Laaksonen, J. Chem. Phys. 111, 7519 (1999).

${ }^{10}$ A. Warshel and M. Levitt, J. Mol. Biol. 103, 227 (1976).

${ }^{11}$ U. C. Singh and P. A. Kollman, J. Comput. Chem. 7, 718 (1986).

${ }^{12}$ M. J. Field, P. A. Bash, and M. Karplus, J. Comput. Chem. 11, 700 (1990).

${ }^{13}$ J. Gao, P. Amara, C. Alhambra, and M. J. Field, J. Phys. Chem. A 102, 4714 (1998).

${ }^{14}$ M. Eichinger, P. Tavan, J. Hutter, and M. Parrinello, J. Chem. Phys. 110, 10452 (1999).

${ }^{15}$ M. C. Colombo, L. Guidoni, A. Laio et al., Chimia 56, 13 (2002).

${ }^{16}$ I. Antes and W. Thiel, J. Phys. Chem. A 103, 9290 (1999).

${ }^{17}$ Y. Zhang, T. -S. Lee, and W. Yang, J. Chem. Phys. 110, 46 (1999). 
${ }^{18}$ N. Reuter, A. Dejaegere, B. Maigret, and M. Karplus, J. Phys. Chem. A 104, 1720 (2000).

${ }^{19}$ G. Monard, M. Loos, V. Thery, K. Baka, and J.-L. Rivail, Int. J. Quantum Chem. 58, 153 (1996).

${ }^{20}$ V. Kairys and J. Jensen, J. Phys. Chem. A 104, 6656 (2000).

${ }^{21}$ R. Murphy, D. Philipp, and R. Friesner, J. Comput. Chem. 21, 1442 (2000)

${ }^{22}$ D. Das, K. P. Eurenius, E. M. Billings, P. Sherwood, D. C. Chatfield, M. Hodoscek, and B. R. Brooks, J. Chem. Phys. 117, 10534 (2002).

${ }^{23} \mathrm{~A}$. Kohlmeyer (private communication).

${ }^{24}$ To communicate with CPMD (Ref. 26) we develop the interface code so as to hook with the existing EGO (Ref. 14) interface program implemented in CPMD v3.9.1 without any interference to either EGO or CPMD. In the interface, we use some of the open source functions distributed in EGO-VIII (Ref. 14) release and one of the functions provided by Groenhof et al. (Ref. 48) in connection with their GROMACS QM/MM interface with other QM codes.

${ }^{25}$ E. Lindahl, B. Hess, and D. van der Spoel, J. Mol. Model. 7, 306 (2001).

${ }^{26}$ R. Car and M. Parrinello, Phys. Rev. Lett. 55, 2471 (1985).

${ }^{27}$ D. A. McQuarrie, Statistical Mechanics (University Science Books, Sausalito, CA, 2000), p. 234.

${ }^{28}$ D. J. Griffiths, Introduction to Quantum Mechanics, 2nd ed. (Upper Saddle River, NJ, 2005), p. 403.

${ }^{29}$ I. N. Levine, Quantum Chemistry, 5th ed. (Prentice-Hall, Upper Saddle River, NJ, 2000), p. 135.

${ }^{30}$ P. K. Biswas, Eur. Phys. J. D 29, 321 (2002).

${ }^{31}$ J. Nocedal, Math. Comput. 35, 773 (1980).

${ }^{32}$ A. D. Becke and K. E. Edgrcombe, J. Chem. Phys. 92, 5397 (1990).
${ }^{33}$ We use the key word GROMACS-CPMD to refer to the QM/MM calculation performed with GROMACS v3.2.1 (Ref. 25) and CPMD v3.9.1 (Ref. 26).

${ }^{34}$ J. A. Odutola and T. R. Dyke, J. Chem. Phys. 72, 5062 (1980).

${ }^{35} \mathrm{~J}$. B. Hasted, in Water: A Comprehensive Treatise, edited by F. Franks (Plenum, New York, 1972), Vol. 1, pp. 255-309.

${ }^{36}$ K. Ichikawa, Y. Kameda, T. Yamaguchi, H. Wakita, and M. Misawa, Mol. Phys. 73, 79 (1991).

${ }^{37}$ M. J. Frisch, G. W. Trucks, H. B. Schlegel et al. GAUSSIAN 98 Gaussian Inc., Pittsburgh, PA, 1998.

${ }^{38}$ W. L. Jorgensen, J. Chandrasekhar, and J. D. Madura, J. Chem. Phys. 79, 926 (1983).

${ }^{39}$ N. Troullier and J. L. Martins, Phys. Rev. B 43, 1993 (1991).

${ }^{40}$ S. Goedecker, J. Hutter, and M. Teter, Phys. Rev. B 54, 1703 (1996).

${ }^{41}$ M. W. Mahoney and W. L. Jorgensen, J. Chem. Phys. 112, 8910 (2000).

${ }^{42}$ W. Humphrey, A. Dalke, and K. Schulten, J. Mol. Graphics 14, 33 (1996).

${ }^{43}$ W. L. Jorgensen and J. Tirado-Rives, J. Am. Chem. Soc. 110, 1657 (1988).

${ }^{44}$ K. Laasonen, A. Pasquarello, C. Lee, R. Car, and D. Vanderbilt, Phys. Rev. B 47, 10142 (1993); source: http://www.physics.rutgers.edu/dhv/ uspp/

${ }^{45}$ C. Rovira, J. Phys.: Condens. Matter 15, S1809 (2003).

${ }^{46}$ S. -M. Peng and J. A. Ibers, J. Am. Chem. Soc. 98, 8032 (1976).

${ }^{47}$ J. Vojtechovsky, K. Chu, J. Berendzen, R. M. Sweet, and I. Schlichting, Biophys. J. 77, 2153 (1999).

${ }^{48}$ G. Groenhof, M. Bouxin-Cademartory, B. Hess, S. P. de Visser, H. J. C. Berendsen, M. Olivucci, A. E. Mark, and M. A. Robb, J. Am. Chem. Soc. 126, 4228 (2004). 